\title{
Fourier Coefficients of a Class of Eta Quotients of Weight 4
}

\author{
Barış Kendirli * \\ Department of Mathematics, Istanbul Aydin University, Turkey \\ *Corresponding author E-mail: bariskendirli@aydin.edu.tr
}

\begin{abstract}
Recently, there have been several works on the coefficients of the Fourier series expansions of a class of eta quotients by Williams, Yao, $\mathrm{Xia}$ and Jin, Kendirli, and Alaca. Some important explicit formulas have been discovered. Williams expressed all coefficients of one hundred and twenty-six eta quotients in terms of $\sigma(n), \sigma\left(\frac{n}{2}\right), \sigma\left(\frac{n}{3}\right)$ and $\sigma\left(\frac{n}{6}\right)$, and Yao, Xia and Jin, following the method of proof of Williams, expressed only even coefficients of one hundred and four eta quotients in terms of $\sigma_{3}(n), \sigma_{3}\left(\frac{n}{2}\right), \sigma_{3}\left(\frac{n}{3}\right)$ and $\sigma_{3}\left(\frac{n}{6}\right)$. The author has expressed the even and odd coefficients of the Fourier series expansions of a class of eta quotients in terms of $\sigma_{k-1}(n), \sigma_{k-1}\left(\frac{n}{2}\right), \sigma_{k-1}\left(\frac{n}{3}\right)$ and $\sigma_{k-1}\left(\frac{n}{6}\right)$ for $k=6,8,10,12,14,16,18,20,22,24$. Meanwhile, Alaca has obtained the coefficients of the Fourier series expansions of a class of eta quotients in $M_{2}\left(\Gamma_{0}, \chi\right)$ in terms of $\sigma(n), \sigma\left(\frac{n}{2}\right), \sigma\left(\frac{n}{3}\right)$ and $\sigma\left(\frac{n}{6}\right)$. Here, we will express the coefficients of the Fourier series expansions of a class of eta quotients in $M_{4}\left(\Gamma_{0}, \chi\right)$ in terms of $\sigma_{3}(n), \sigma_{3}\left(\frac{n}{2}\right), \sigma_{3}\left(\frac{n}{3}\right), \sigma_{3}\left(\frac{n}{6}\right)$ and Fourier coefficients of the four eta quotients.
\end{abstract}

Keywords: Dedekind eta function; Eisenstein series; Eta quotients; Fourier coefficients; Modular forms.

\section{Introduction}

The divisor function $\sigma_{\mathrm{i}}(\mathrm{n})$ is defined for a positive integer $\mathrm{i}$ by

$\sigma_{\mathrm{i}}(\mathrm{n}):=\sum_{\mathrm{d} \text { positive integer, } \mathrm{d} \mid \mathrm{n}} \mathrm{d}^{\mathrm{i}}$, if $\mathrm{n}$ is a positive integer, and

$\sigma_{\mathrm{i}}(\mathrm{n}):=0$, if $\mathrm{n}$ is not a positive integer.

The Dedekind eta function is defined by

$\eta(z):=q^{1 / 24} \prod_{n=1}^{\infty}\left(1-q^{n}\right)$,

where

$\mathrm{q}:=\mathrm{e}^{2 \pi \mathrm{iz}}, \mathrm{z} \in \mathrm{H}=\{\mathrm{x}+\mathrm{iy}: \mathrm{y}>0\}$

and, an eta quotient of level $\mathrm{n}$ is defined by

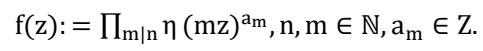

There have been considerable effort to determine explicit formulas for the Fourier coefficients of eta quotients since they are the building blocks of modular forms of level $\mathrm{n}$ and weight $\mathrm{k}$. The book of Köhler [1] (Chapter 3, pg.39) describes such expansions by means of Hecke Theta series, and it develops algorithms for the determination of suitable eta quotients. One can find more information in [2], [3], [4], [5] and [6]. The author has also determined the Fourier coefficients of the theta series associated with some quadratic forms, see [8], [9], [10], [11], [12] and [13]. Recently, Williams [14] discovered explicit formulas for the coefficients of Fourier series expansions of a class of one hundred and twenty-six eta quotients in terms of $\sigma(\mathrm{n}), \sigma\left(\frac{\mathrm{n}}{2}\right), \sigma\left(\frac{\mathrm{n}}{3}\right)$ and $\sigma\left(\frac{\mathrm{n}}{6}\right)$. $\frac{\eta^{2}(2 z) \eta^{4}(4 z) \eta^{6}(6 z)}{\eta^{2}(z) \eta^{2}(3 z) \eta^{4}(12 z)}=1+\sum_{n=1}^{\infty} c(n) q^{n}$

where

$\mathrm{c}(\mathrm{n})=2 \sigma(\mathrm{n})-3 \sigma(\mathrm{n} / 2)+4 \sigma(\mathrm{n} / 4)+9 \sigma(\mathrm{n} / 6)-36 \sigma(\mathrm{n} / 12)$.

Then Yao, Xia and Jin [15] expressed the even Fourier coefficients of one hundred and four eta quotients in terms of $\sigma_{3}(\mathrm{n}), \sigma_{3}\left(\frac{\mathrm{n}}{2}\right), \sigma_{3}\left(\frac{\mathrm{n}}{3}\right)$ and $\sigma_{3}\left(\frac{\mathrm{n}}{6}\right)$. One example is as follows:

$\frac{\eta^{25}(2 z) \eta^{4}(3 z)}{\eta^{12}(z) \eta^{5}(4 z) \eta^{3}(6 z) \eta(12 z)}=1+\sum_{n=1}^{\infty} \mathrm{c}(n) q^{n}$,

where

$c(2 n)=65 \sigma_{3}(n)-68 \sigma_{3}(n / 2)-81 \sigma_{3}(n / 3)+324 \sigma_{3}(n / 6)$.

Motivated by these two results, we find that we can express the even and odd coefficients of the Fourier series expansions of a class of eta quotients in terms of $\sigma_{\mathrm{k}-1}(\mathrm{n}), \sigma_{\mathrm{k}-1}\left(\frac{\mathrm{n}}{2}\right), \sigma_{\mathrm{k}-1}\left(\frac{\mathrm{n}}{3}\right)$ and $\sigma_{\mathrm{k}-1}\left(\frac{\mathrm{n}}{6}\right)$ for $\mathrm{k}=6[16], 8[17], 10[18], 12[19], 14[20], 16[21]$, $18[22], 20[23], 22[24], 24[25]$. Meanwhile, Alaca [26] has obtained the coefficients of the Fourier series expansions of a class of eta quotients in $\mathrm{M}_{2}\left(\Gamma_{0}, \chi\right)$ in terms of $\sigma(\mathrm{n}), \sigma\left(\frac{\mathrm{n}}{2}\right), \sigma\left(\frac{\mathrm{n}}{3}\right)$ and $\sigma\left(\frac{\mathrm{n}}{6}\right)$. Here, we will express the coefficients of the Fourier series expansions of a class of one hundred and fifty-six eta quotients in $\mathrm{M}_{4}\left(\Gamma_{0}, \chi\right)$ in terms of $\sigma_{3}(\mathrm{n}), \sigma_{3}\left(\frac{\mathrm{n}}{2}\right), \sigma_{3}\left(\frac{\mathrm{n}}{3}\right), \sigma_{3}\left(\frac{\mathrm{n}}{6}\right)$ and the Fourier coefficients of the four eta quotients.

Here we give the following Lemma, see[ [27] Theorem 1.64] about the modularity of an eta quotient. 
Lemma 1. An eta quotient of level $\mathrm{N}$ is a meromorphic modular form of weight $\frac{1}{2} \sum_{\mathrm{m} \mid \mathrm{N}} \mathrm{a}_{\mathrm{m}}$ on $\Gamma_{0}(\mathrm{~N})$, with Dirichlet character $\chi$, having rational coefficients with respect to $q$ if

a) $\sum_{\mathrm{m} \mid \mathrm{N}} \mathrm{a}_{\mathrm{m}}$ is even,

b) $\sum_{\mathrm{m} \mid \mathrm{N}} \mathrm{ma}_{\mathrm{m}} \equiv \sum_{\mathrm{m} \mid \mathrm{N}} \frac{\mathrm{N}}{\mathrm{m}} \mathrm{a}_{\mathrm{m}} \equiv 0 \bmod 24$,

c) $\chi(\mathrm{m})=\left(\frac{(-1)^{\mathrm{k}} \prod_{\mathrm{m} \mid \mathrm{N}} \mathrm{m}^{\mathrm{a} m}}{\mathrm{~m}}\right)$.

In particular, since

$2^{a_{2}} 3^{a_{3}} 4^{a_{4}} 6^{a_{6}} 12^{a_{12}}=2^{a_{2}+2 a_{4}+a_{6}+2 a_{12}} 3^{a_{3}+a_{6}+a_{12}}$

an eta quotient of level 12 is a meromorphic modular form of weight 4 if

$a_{1}+a_{2}+a_{3}+a_{4}+a_{6}+a_{12}=8$

and

$\mathrm{a}_{1}+2 \mathrm{a}_{2}+3 \mathrm{a}_{3}+4 \mathrm{a}_{4}+6 \mathrm{a}_{6}+12 \mathrm{a}_{12} \equiv 12 \mathrm{a}_{1}+6 \mathrm{a}_{2}+4 \mathrm{a}_{3}+3 \mathrm{a}_{4}+$ $2 \mathrm{a}_{6}+\mathrm{a}_{12} \equiv 0 \bmod 24$

Since $a_{2}+a_{6}$ is an even integer, we conclude that it is a meromorphic modular form iff $\mathrm{a}_{3}+\mathrm{a}_{6}+\mathrm{a}_{12}$ is an even integer, and it is a meromorphic modular form with $\chi_{3}$ iff $a_{3}+a_{6}+a_{12}$ is an odd integer, where $\chi_{3}$ is the unique primitive Dirichlet character mod12. On the other hand, the modular forms are holomorphic iff its order at cusps, $\frac{1}{1}, \frac{1}{2}, \frac{1}{3}, \frac{1}{4}, \frac{1}{6}$ and $\frac{1}{12}$ are nonnegative, see [1] i.e.,

$\sum_{\mathrm{m} \mid 12} \frac{(\operatorname{gcd}(c, \mathrm{~m}))^{2}}{\mathrm{~m}} \mathrm{a}_{\mathrm{m}} \geq 0$ for $\mathrm{c} \mid 12$.

Theorem 1: Let $\chi_{0}$ be the trivial character mod 1, i.e., it sends $n$ to $1, \chi_{1}$ be the primitive Dirichlet character $\bmod 4$, and $\chi_{2}$ is the primitive Dirichlet character mod 3. Then the Eisenstein subspace of $M_{4}\left(\Gamma_{0}(12), \chi_{3}\right)$ is generated by

$\mathrm{E}_{4}^{\chi_{1}, \chi_{2}}=\sum_{\mathrm{n}=1}^{\infty}\left(\sum_{0<\mathrm{d} \mid \mathrm{n}} \chi_{1}\left(\frac{\mathrm{n}}{\mathrm{d}}\right) \chi_{2}(\mathrm{~d}) \mathrm{d}^{3}\right) \mathrm{q}^{\mathrm{n}}=\mathrm{q}-\mathrm{q}^{2}+41 \mathrm{q}^{3}+113 \mathrm{q}^{4}-$ $124 q^{5}+155 q^{6}+874 q^{7}+0\left(q^{8}\right)$,

$E_{4}^{\chi_{2}, \chi_{1}}=\sum_{n=1}^{\infty}\left(\sum_{0<\mathrm{d} \mid \mathrm{n}} \chi_{2}\left(\frac{\mathrm{n}}{\mathrm{d}}\right) \chi_{1}(\mathrm{~d}) \mathrm{d}^{3}\right) \mathrm{q}^{\mathrm{n}}=\mathrm{q}-\mathrm{q}^{2}-27 \mathrm{q}^{3}+\mathrm{q}^{4}+$ $124 q^{5}+27 q^{6}-342 q^{7}+O\left(q^{8}\right)$

$\mathrm{E}_{4}^{\chi_{0}, \chi_{3}}=-\frac{\mathrm{B}_{4, \chi_{3}}}{8}+\sum_{\mathrm{n}=1}^{\infty}\left(\sum_{0<\mathrm{d} \mid \mathrm{n}} \chi_{0}\left(\frac{\mathrm{n}}{\mathrm{d}}\right) \chi_{3}(\mathrm{~d}) \mathrm{d}^{3}\right) \mathrm{q}^{\mathrm{n}}=23+\mathrm{q}+\mathrm{q}^{2}+\mathrm{q}^{3}+$ $q^{4}-124 q^{5}+q^{6}-342 q^{7}+0\left(q^{8}\right)$,

$\mathrm{E}_{4}^{\chi_{3}, \chi_{0}}=\sum_{\mathrm{n}=1}^{\infty}\left(\sum_{0<\mathrm{d} \mid \mathrm{n}} \chi_{3}\left(\frac{\mathrm{n}}{\mathrm{d}}\right) \chi_{0}(\mathrm{~d}) \mathrm{d}^{3}\right) \mathrm{q}^{\mathrm{n}}=\mathrm{q}+8 \mathrm{q}^{2}+27 \mathrm{q}^{3}+64 \mathrm{q}^{4}+$ $124 q^{5}+216 q^{6}+342 q^{7}+O\left(q^{8}\right)$

and, the cuspidal subspace $S_{4}\left(\Gamma_{0}(12), \chi_{3}\right)$ is generated by

$f_{1}:=\sum_{n=0}^{\infty} f_{1}(n) q^{n}=\frac{\eta^{2}(2 z) \eta^{10}(3 z) \eta(4 z) \eta(12 z)}{\eta^{2}(z) \eta^{4}(6 z)}$,

$f_{2}:=\sum_{n=0}^{\infty} f_{2}(n) q^{n}=\frac{\eta^{2}(2 z) \eta^{7}(3 z) \eta^{4}(12 z)}{\eta(z) \eta^{4}(6 z)}$

$f_{3}:=\sum_{n=0}^{\infty} f_{3}(n) q^{n}=\frac{\eta^{4}(z) \eta^{7}(4 z) \eta^{2}(6 z)}{\eta^{4}(2 z) \eta(12 z)}$

$f_{4}:=\sum_{n=0}^{\infty} f_{4}(n) q^{n}=\frac{\eta^{4}(z) \eta^{2}(4 z) \eta^{7}(6 z)}{\eta^{3}(2 z) \eta^{2}(12 z)}$

Proof:

Since

$\chi_{3}=\chi_{0} * \chi_{3}, \chi_{3}=\chi_{3} * \chi_{0}, \chi_{3}=\chi_{1} * \chi_{2}, \chi_{3}=\chi_{2} * \chi_{1}$, the Eisenstein subspace of $\mathrm{M}_{4}\left(\Gamma_{0}(12), \chi_{3}\right)$ is generated by $\mathrm{E}_{4}^{\chi_{1}, \chi_{2}}, \mathrm{E}_{4}^{\chi_{2}, \chi_{1}}, \mathrm{E}_{4}^{\chi_{0}, \chi_{3}}$ and $\mathrm{E}_{4}^{\chi_{3}, \chi_{0}}$.

Since $f_{1}, f_{2}, f_{3}$ and $f_{4}$ are in $S_{4}\left(\Gamma_{0}(12), \chi_{3}\right)$, linearly independent and $\operatorname{dim}\left(\mathrm{S}_{4}\left(\Gamma_{0}(12), \chi_{3}\right)\right)=4$, the result follows. Let

$\delta\left(\mathrm{b}_{1}\right)=\left\{\begin{array}{l}0 \text { if } \mathrm{b}_{1} \neq 0 \\ 1 \text { if } \mathrm{b}_{1}=0\end{array}\right.$

Now we can state our main Conclusion:

Theorem 2: The coefficients of the Fourier series of one hundred and fifty-six eta quotients

$\eta^{\mathrm{a}_{1}}(\mathrm{z}) \eta^{\mathrm{a}_{2}}(2 \mathrm{z}) \eta^{\mathrm{a}_{3}}(3 \mathrm{z}) \eta^{\mathrm{a}_{4}}(4 \mathrm{z}) \eta^{\mathrm{a}_{6}}(6 \mathrm{z}) \eta^{\mathrm{a}_{12}}(12 \mathrm{z})=\delta\left(\mathrm{b}_{1}\right)+\sum_{\mathrm{n}=1}^{\infty} \mathrm{c}(\mathrm{n}) \mathrm{q}^{\mathrm{n}}$ in $\mathrm{M}_{4}\left(\Gamma_{0}(12), \chi_{3}\right)$ are given in the form

$\mathrm{c}_{1} \sigma_{3}(\mathrm{n})+\mathrm{c}_{2} \sigma_{3}\left(\frac{\mathrm{n}}{2}\right)+\mathrm{c}_{3} \sigma_{3}\left(\frac{\mathrm{n}}{3}\right)+\mathrm{c}_{4} \sigma_{3}\left(\frac{\mathrm{n}}{4}\right)+\mathrm{c}_{6} \sigma_{3}\left(\frac{\mathrm{n}}{6}\right)+\mathrm{c}_{12} \sigma_{3}\left(\frac{\mathrm{n}}{12}\right)$

$+r_{1} f_{1}(n)+r_{2} f_{2}(n)+r_{3} f_{3}(n)+r_{4} f_{4}(n)$

As in the Table in Appendix.

Remark 1: The coefficients of the Fourier series of the 104 eta quotients in $M_{4}\left(\Gamma_{0}(12)\right)$ are given in the form

$\mathrm{c}_{1} \sigma_{3}(\mathrm{n})+\mathrm{c}_{2} \sigma_{3}\left(\frac{\mathrm{n}}{2}\right)+\mathrm{c}_{3} \sigma_{3}\left(\frac{\mathrm{n}}{3}\right)+\mathrm{c}_{4} \sigma_{3}\left(\frac{\mathrm{n}}{4}\right)+\mathrm{c}_{6} \sigma_{3}\left(\frac{\mathrm{n}}{6}\right) \mathrm{c}_{12} \sigma_{3}\left(\frac{\mathrm{n}}{12}\right)$

$+r_{1} g_{1}(n)+r_{2} g_{2}(n)+r_{3} h(n), g_{1}(2 n)=g_{2}(2 n)=h(2 n-1)=0$,

exactly as in [15]. But our method is obviously much simpler than it since we didn't need to use $(\mathrm{p}, \mathrm{k})$ parametrizations.

Remark 2: $S_{4}\left(\Gamma_{0}(12), \chi_{3}\right)$ is 4 dimensional, see [28] (Chapter 3, pg.87 and Chapter 5, pg.197), and it is generated by $\Delta_{s}$, and its three conjugates, where $t=\sqrt{3}, s=\sqrt{-140+80 \sqrt{3}}$ and

$\Delta_{\mathrm{s}}=\mathrm{q}+\frac{1}{160}\left(-\mathrm{s}^{3}+2 \mathrm{~s}^{2}-300 \mathrm{~s}+280\right) \mathrm{q}^{2}+\frac{1}{80}\left(\mathrm{~s}^{3}-2 \mathrm{~s}^{2}+260 \mathrm{~s}-\right.$ $280) q^{3}+\frac{1}{40}\left(-s^{3}-260 s-80\right) q^{4}+\frac{1}{40}\left(s^{3}+300 s\right) q^{5}+\frac{1}{160}\left(7 s^{3}+\right.$ $\left.10 s^{2}+1940 s+440\right) q^{6}+\frac{1}{40}\left(-s^{3}-260 s\right) q^{7}+\frac{1}{40}\left(-s^{3}-6 s^{2}-300 s-\right.$ $840) q^{8}+\frac{1}{40}\left(-3 s^{3}-900 s-120\right) q^{9}+\frac{1}{20}\left(s^{3}+260 s+400\right) q^{10}+$ $\frac{1}{4}\left(s^{2}+140\right) q^{11}+O\left(q^{12}\right)$.

Here, $\mathrm{s},-\mathrm{s},(4 * \mathrm{t}+7) * \mathrm{~s}$ and $-(4 * \mathrm{t}+7) * \mathrm{~s}$ are distinct four conjugate roots of the irreducible polynomial $x^{4}+280 x^{2}+400$. By simple calculation, we see that

$\mathrm{f}_{1}=\left(\frac{1}{120}(7 \mathrm{t}+15) \mathrm{s}+\frac{1}{12}(-\mathrm{t}+3)\right) \Delta_{\mathrm{s}}(\mathrm{z})+\left(\frac{1}{120}(-7 \mathrm{t}-15) \mathrm{s}+\frac{1}{12}(-\mathrm{t}+\right.$

3)) $\Delta_{-s}(\mathrm{z})+\left(\frac{1}{120}(11 \mathrm{t}+21) \mathrm{s}+\frac{1}{12}(\mathrm{t}+3)\right) \Delta_{(4 * \mathrm{t}+7) * \mathrm{~s}}(\mathrm{z})+\left(\frac{1}{120}(-11 \mathrm{t}-\right.$ 21) $\left.s+\frac{1}{12}(t+3)\right) \Delta_{-(4 * t+7) * s}(z)$,

$\mathrm{f}_{2}=\left(\frac{1}{80}(3 \mathrm{t}+6) \mathrm{s}-\frac{1}{24} \mathrm{t}\right) \Delta_{\mathrm{s}}(\mathrm{z})+\left(\frac{1}{80}(-3 \mathrm{t}-6) \mathrm{s}-\frac{1}{24} \mathrm{t}\right) \Delta_{-s}(\mathrm{z})+$ $\left(\frac{1}{80}(3 \mathrm{t}+6) \mathrm{s}+\frac{1}{24} \mathrm{t}\right) \Delta_{(4 * \mathrm{t}+7) * \mathrm{~s}}(\mathrm{z})+\left(\frac{1}{80}(-3 \mathrm{t}-6) \mathrm{s}+\frac{1}{24} \mathrm{t}\right) \Delta_{-(4 * \mathrm{t}+7) * \mathrm{~s}}(\mathrm{z})$,

$\mathrm{f}_{3}=\left(\frac{1}{80}(-9 \mathrm{t}-16) \mathrm{s}+\frac{1}{8}(-\mathrm{t}+2)\right) \Delta_{\mathrm{s}}(\mathrm{z})+\left(\frac{1}{80}(9 \mathrm{t}+16) \mathrm{s}+\frac{1}{8}(-\mathrm{t}+\right.$ 2)) $\Delta_{-s}(\mathrm{z})+\left(\frac{1}{80}(-\mathrm{t}-4) \mathrm{s}+\frac{1}{8}(\mathrm{t}+2)\right) \Delta_{(4 * \mathrm{t}+7) * \mathrm{~s}}(\mathrm{z})+\left(\frac{1}{80}(\mathrm{t}+4) \mathrm{s}+\right.$ $\left.\frac{1}{8}(\mathrm{t}+2)\right) \Delta_{-(4 * \mathrm{t}+7) * \mathrm{~s}}(\mathrm{z})$,

$\mathrm{f}_{4}=\left(\frac{1}{120}(-8 \mathrm{t}-15) \mathrm{s}+\frac{1}{12}(-2 \mathrm{t}+3)\right) \Delta_{\mathrm{s}}(\mathrm{z})+\left(\frac{1}{120}(8 \mathrm{t}+15) \mathrm{s}+\right.$ $\left.\frac{1}{12}(-2 \mathrm{t}+3)\right) \Delta_{-s}(\mathrm{z})+\left(\frac{1}{120}(-4 \mathrm{t}-9) \mathrm{s}+\frac{1}{12}(2 \mathrm{t}+3)\right) \Delta_{(4 * \mathrm{t}+7) * \mathrm{~s}}(\mathrm{z})+$ $\left(\frac{1}{120}(4 t+9) s+\frac{1}{12}(2 t+3)\right) \Delta_{-(4 * t+7) * s}(z)$. 


\section{References}

[1] G. Köhler, Eta Products and Theta Series Identities, SpringerVerlag, Berlin, 2011. http://dx.doi.org/10.1007/978-3-642-16152-0.

[2] B. Gordon, Some identities in combinatorial analysis, Quart. J. Math. Oxford Ser.12 (1961), 285-290.

[3] V. G. Kac, Infinite-dimensional algebras, Dedekind's $\eta$-function, classical Möbius function and the very strange formula, Adv. Math. 30 (1978), 85-136. http://dx.doi.org/10.1016/0001-8708(78)90033$\underline{6}$.

[4] I. G. Macdonald, Affine root systems and Dedekind's $\eta$-function, Invent. Math. 15 (1972), 91-143. http://dx.doi.org/10.1007/BF01418931.

[5] I. J. Zucker, A systematic way of converting infinite series into infinite products, J. Phys. A 20 (1987) L13-L17. http://dx.doi.org/10.1088/0305-4470/20/1/003.

[6] I. J. Zucker, Further relations amongst infinite series and products:II. The evaluation of three-dimensional lattice sums, J. Phys. A 23 (1990), 117-132. http://dx.doi.org/10.1088/03054470/23/2/009.

[7] B. Gordon and S. Robins, Lacunarity of Dedekind $\eta$-products, Glasgow Math. J. 37 (1995), 1-14 http://dx.doi.org/10.1017/S0017089500030329.

[8] B. Kendirli, Evaluation of Some Convolution Sums by Quasimodular Forms, European Journal of Pure and Applied Mathematics ISSN 13075543 Vol.8, No. 1 (2015), 81-110.

[9] B. Kendirli, Evaluation of Some Convolution Sums and Representation Numbers of Quadratic Forms of Discriminant 135 , British Journal of Mathematics and Computer Science, Vol. 66 (2015), 494-531. http://dx.doi.org/10.9734/BJMCS/2015/13973.

[10] B. Kendirli, Evaluation of Some Convolution Sums and the Representation numbers, Ars Combinatoria Vol. CXVI (2014), 65 91.

[11] B. Kendirli, Cusp Forms in $S_{4}\left(\Gamma_{0}(79)\right)$ and the number of representations of positive integers by some direct sum of binary quadratic forms with discriminant -79, Bulletin of the Korean Mathematical Society, Vol. $49 \quad 3$ (2012), 529-572. http://dx.doi.org/10.4134/BKMS.2012.49.3.529.

[12] B. Kendirli, Cusp Forms in $S_{4}\left(\Gamma_{0}(47)\right)$ and the number of representations of positive integers by some direct sum of binary quadratic forms with discriminant -47 , International Journal of Mathematics and Mathematical Sciences Vol. (2012), Article ID 303492, 10 pages.

[13] B. Kendirli, The Bases of $M_{4}\left(\Gamma_{0}(71)\right), M_{6}\left(\Gamma_{0}(71)\right)$ and the Number of Representations of Integers, Mathematical Problems in Engineering Vol. (2013), Article ID 695265, 34 pages.
[14] K. S. Williams, Fourier series of a class of eta quotients, Int. J. $\begin{array}{llll}\text { Number Theory } 8 & \text { (2012), } & \text { 993-1004. }\end{array}$ http://dx.doi.org/10.1142/S1793042112500595.

[15] Olivia X. M. Yao, Ernest X. W. Xia and J. Jin, Explicit Formulas for the Fourier coefficients of a class of eta quotients, International Journal of Number Theory Vol. 922 (2013), 487-503. http://dx.doi.org/10.1142/S179304211250145X

[16] B. Kendirli, Fourier Coefficients of a Class of Eta Quotients, INTEGERS (to be published) (2016).

[17] B. Kendirli, Fourier Coefficients of Some Eta Quotients of Weight 8, European Journal of Pure and Applied Mathematics Vol. 8, No. $3,(2015), 395-416$

[18] B. Kendirli, Fourier Coefficients of a Class of Eta Quotients of Weight 10, J. Math. Comput. Sci. 5 (2015), No. 6, 780-810.

[19] B. Kendirli, Fourier Coefficients of a Class of Eta Quotients of Weight 12, British Journal of Mathematics and Computer Science, $\begin{array}{lllll}\text { Vol. } & 10 & 2 & \text { (2015), }\end{array}$ http://dx.doi.org/10.9734/BJMCS/2015/18442.

[20] B. Kendirli, Fourier Coefficients of a Class of Eta Quotients of Weight 14 with Level 12, Journal of Basic\&Applied Sciences (2015), 11, 454-483. $\quad$ http://dx.doi.org/10.6000/19275129.2015.11.64

[21] B. Kendirli, Fourier Coefficients of a Class of Eta Quotients of Weight 16 with Level 12 (2015), Applied Mathematics 6, quotients, International Journal of Number Theory Vol. 92 (2013), 487-503.

[22] B. Kendirli, Fourier Coefficients of a Class of Eta Quotients of Weight 18 with Level 12, Pure and Applied Mathematics Journal (2015), 4(4) http://dx.doi.org/10.11648/j.pamj.20150404.17.

[23] B. Kendirli, Fourier Coefficients of a Class of Eta Quotients of Weight 20 with Level 12, International Journal of Advanced Mathematical Sciences, 3 (2) (2015), 121-146. http://dx.doi.org/10.14419/ijams.v3i2.5247.

[24] B. Kendirli, Fourier Coefficients of a Class of Eta Quotients of Weight 22 with Level 12, European Journal of Mathematics and Computer Science, Vol.2, No.1, (2015), 1-47. http://dx.doi.org/10.9734/BJMCS/2015/18442.

[25] B. Kendirli, Fourier Coefficients of a Class of Eta Quotients of Weight 24 with Level 12, International Journal of Electronics, Mechanical and Mechatronics Engineering (to be published) (2016).

[26] A. Alaca, S. Alaca and Z. S. Aygin, Fourier Coefficients of a class of eta quotients of weight 2 Int. J. Number Theory 8 (2015).

[27] K. Ono, The Web of Modularity: Arithmetic of the Coefficients of Modular Forms and q-series, CBMS Regional Conf. Series in Math. 102, Amer. Math. Soc., Providence, R. I.,2004.

[28] F. Diamond, J. Shurman, A First Course in Modular Forms, Springer Graduate Texts in Mathematics 228, 2005

\section{Appendix}

Table

\begin{tabular}{|c|c|c|c|c|c|c|c|c|c|c|c|c|c|c|}
\hline No & $a_{1}$ & $a_{2}$ & $a_{3}$ & $a_{4}$ & $a_{6}$ & $a_{12}$ & $c_{1}$ & $c_{2}$ & $c_{3}$ & $c_{4}$ & $r_{1}$ & $r_{2}$ & $r_{3}$ & $r_{4}$ \\
\hline 1 & -10 & 20 & 10 & -9 & -10 & 7 & $\frac{16}{23}$ & 0 & 0 & $-\frac{1}{23}$ & 0 & $-\frac{166}{23}$ & & $-\frac{27}{23}$ \\
\hline 2 & -9 & 16 & 7 & -2 & -6 & 2 & $\frac{36}{23}$ & 0 & 0 & $0^{23}$ & & $-\frac{205}{92}$ & $-\frac{9}{23}$ & ${\frac{77^{23}}{184}}^{23}$ \\
\hline 3 & -9 & 20 & -1 & -4 & 2 & 0 & $\frac{27}{23}$ & 0 & $\frac{1}{23}$ & 0 & & & $\frac{16}{23}^{23}$ & $-\frac{21}{46}$ \\
\hline 4 & -8 & 14 & 4 & 1 & -4 & 1 & $\frac{27}{23}$ & 0 & 0 & 0 & & & & \\
\hline 5 & -8 & 9 & 12 & 2 & -5 & -2 & & $\frac{1}{23}$ & 0 & 0 & & $\frac{282}{23}$ & $-\frac{40}{23}$ & \\
\hline 6 & -7 & 10 & 1 & 8 & 0 & -4 & $\frac{725}{276}$ & 0 & $\frac{9}{92}$ & $\frac{7}{69}$ & $\frac{3769}{736}$ & $-\frac{3371}{368}$ & $-\frac{51}{92}$ & $-\frac{289}{736}$ \\
\hline 7 & -7 & 13 & 9 & -7 & -9 & 9 & $\frac{8}{69}$ & 0 & 0 & & & -2 & & $-\frac{19}{46}$ \\
\hline 8 & -7 & 17 & 1 & -9 & -1 & 7 & $\frac{2}{23}$ & 0 & 0 & $\frac{\frac{1}{23}}{3}$ & $-\frac{43}{184}$ & & $\begin{array}{r}-\frac{5}{23} \\
48\end{array}$ & $\frac{59}{184}$ \\
\hline 10 & -7 & 18 & 9 & -8 & -8 & -3 & $\begin{array}{l}0 \\
\underline{72} \\
\end{array}$ & $\begin{array}{l}0 \\
1 \\
\end{array}$ & 0 & $\begin{array}{l}\overline{23} \\
0\end{array}$ & $\begin{array}{l}-\overline{23} \\
221 \\
\end{array}$ & $\begin{array}{l}\overline{23} \\
-244 \\
\end{array}$ & $\begin{array}{r}-\overline{23} \\
-\frac{34}{4} \\
\end{array}$ & \\
\hline 11 & -7 & 8 & 9 & 0 & 2 & -4 & & $\begin{array}{l}23 \\
1 \\
\end{array}$ & 0 & 0 & $\begin{array}{r}46 \\
118 \\
\end{array}$ & $\begin{array}{r}23 \\
-216 \\
\end{array}$ & $\begin{array}{r}23 \\
-32 \\
\end{array}$ & \\
\hline 12 & -6 & 9 & 6 & 0 & -5 & 1 & $\begin{array}{r}23 \\
6 \\
\end{array}$ & $\begin{array}{l}23 \\
0\end{array}$ & 0 & 0 & $\begin{array}{r}23 \\
-113 \\
\end{array}$ & $\begin{array}{r}23 \\
-53 \\
\end{array}$ & $\begin{array}{r}23 \\
-\quad 3 \\
\end{array}$ & $\begin{array}{r}23 \\
41 \\
\end{array}$ \\
\hline 12 & -0 & 12 & 2 & 2 & -5 & 4 & $\begin{array}{c}\overline{23} \\
9 \\
\end{array}$ & 0 & 0 & 0 & & $\begin{array}{l}167 \\
184\end{array}$ & $9^{\overline{46}}$ & $\begin{array}{l}\overline{368} \\
-\quad 19 \\
\end{array}$ \\
\hline 14 & -6 & 16 & -2 & -5 & -6 & 3 & $\begin{array}{l}\overline{46} \\
390 \\
\end{array}$ & 0 & $\begin{array}{l}\overline{46} \\
7 \\
\end{array}$ & 66 & $\begin{array}{r}368 \\
-\quad 1288 \\
\end{array}$ & $\begin{array}{l}\overline{184} \\
-13869 \\
\end{array}$ & $\begin{array}{l}\overline{46} \\
-1035 \\
\end{array}$ & $\begin{array}{r}368 \\
-109 \\
\end{array}$ \\
\hline 15 & -6 & 20 & -2 & -7 & 2 & 1 & $\begin{array}{l}29 \\
-806 \\
\end{array}$ & 0 & $\begin{array}{l}87 \\
1 \\
\end{array}$ & $\begin{array}{l}29 \\
-32\end{array}$ & $\begin{array}{r}87 \\
2600 \\
\end{array}$ & $\begin{array}{r}29 \\
29289 \\
\end{array}$ & $3009^{29}$ & $117^{29}$ \\
\hline 16 & -6 & 6 & 6 & 3 & 4 & -5 & & $\begin{array}{l}0 \\
1 \\
\end{array}$ & $\begin{array}{l}87 \\
0\end{array}$ & $0^{-\overline{29}}$ & $\begin{array}{r}87 \\
110 \\
\end{array}$ & $\begin{array}{r}29 \\
-206 \\
\end{array}$ & $\begin{array}{r}29 \\
-28 \\
\end{array}$ & $\begin{array}{l}29 \\
7 \\
\end{array}$ \\
\hline 17 & -6 & 6 & 14 & -3 & -8 & 5 & $\begin{array}{l}23 \\
\frac{64}{207}\end{array}$ & $\begin{array}{l}23 \\
0\end{array}$ & 0 & $-\frac{1}{207}$ & $\begin{array}{l}23 \\
-\frac{5}{23}\end{array}$ & $\begin{array}{r}23 \\
-\frac{32}{23}\end{array}$ & $0^{23}$ & $-\frac{23}{23}$ \\
\hline
\end{tabular}




\begin{tabular}{|c|c|c|c|c|c|c|c|c|c|c|c|c|c|c|}
\hline 18 & -6 & 7 & 6 & & 9 & & $\underline{128}$ & 1 & 0 & $-\frac{1}{1}$ & $\underline{120}$ & $-\underline{224}$ & -64 & $-\frac{4}{-}$ \\
\hline 10 & -0 & 1 & 0 & -2 & 9 & -6 & $\begin{array}{l}\overline{69} \\
18\end{array}$ & $\overline{23}$ & 0 & $-\overline{69}$ & $\begin{array}{r}23 \\
121 \\
\end{array}$ & $\begin{array}{r}23 \\
-251 \\
\end{array}$ & $\begin{array}{r}69 \\
-9 \\
\end{array}$ & $\begin{array}{l}\overline{23} \\
\end{array}$ \\
\hline 19 & -6 & 7 & 6 & 4 & -3 & 0 & 23 & 0 & 0 & 0 & 368 & $-\overline{184}$ & $-\frac{3}{46}$ & $\overline{368}$ \\
\hline 20 & -5 & 7 & 3 & 3 & -3 & 3 & 0 & 0 & 0 & 0 & $\frac{1}{2}$ & 7 & 0 & $\frac{1}{2}$ \\
\hline 21 & -5 & 12 & 3 & 2 & -2 & -2 & $-\frac{1}{69}$ & 0 & $\frac{2}{23}$ & $\frac{7}{69}$ & $\frac{1075}{184}$ & $-\frac{629}{92}$ & $-\frac{9}{23}$ & $-\frac{5}{8}$ \\
\hline 22 & -5 & 5 & 3 & 1 & 11 & -7 & $\frac{32}{22}^{69}$ & $\frac{1}{2}$ & $\begin{array}{l}23 \\
0\end{array}$ & $\begin{array}{l}69 \\
0\end{array}$ & $\begin{array}{l}184 \\
102 \\
\end{array}$ & $\begin{array}{r}92 \\
-196 \\
\end{array}$ & $\begin{array}{r}23 \\
-24 \\
\end{array}$ & $\underline{4}^{8}$ \\
\hline 23 & -5 & 5 & 3 & 7 & -1 & -1 & $\frac{27}{27}$ & 0 & 0 & 0 & $\begin{array}{r}23 \\
423 \\
\end{array}$ & $\begin{array}{r}23 \\
-549 \\
\end{array}$ & $\begin{array}{r}23 \\
-25 \\
\end{array}$ & $\begin{array}{r}23 \\
81 \\
\end{array}$ \\
\hline 24 & -5 & 6 & 3 & 2 & 4 & -2 & $\begin{array}{l}46 \\
12 \\
\end{array}$ & 0 & 0 & 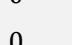 & $\begin{array}{l}736 \\
117 \\
\end{array}$ & $\begin{array}{r}368 \\
-99\end{array}$ & $\begin{array}{r}92 \\
-3 \\
\end{array}$ & $\begin{array}{l}736 \\
\end{array}$ \\
\hline 25 & -5 & 6 & 11 & -4 & -8 & 2 & $\begin{array}{c}23 \\
16 \\
\end{array}$ & 0 & 0 & 1 & 184 & $\begin{array}{r}92 \\
21\end{array}$ & $4_{4}^{23}$ & $\begin{array}{c}184 \\
3\end{array}$ \\
\hline 26 & -4 & 3 & 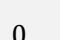 & 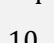 & 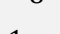 & 0 & $\begin{array}{l}207 \\
81\end{array}$ & 0 & 1 & 207 & $\begin{array}{l}0 \\
549\end{array}$ & $\begin{array}{l}23 \\
639\end{array}$ & $\overline{69}{ }_{39}$ & $\begin{array}{l}23 \\
171\end{array}$ \\
\hline 27 & -4 & $J$ & 0 & 10 & 1 & -2 & $\frac{01}{184}$ & 0 & $\overline{184}$ & 0 & $\frac{549}{736}$ & $-\frac{\overline{368}}{168}$ & $-\overline{92}$ & 736 \\
\hline $\begin{array}{l}27 \\
28\end{array}$ & -4 & 4 & 0 & -1 & 18 & -9 & $\frac{64}{69}$ & $\frac{1}{23}$ & 0 & $\frac{1}{69}$ & $\frac{84}{23}$ & $-\frac{168}{23}$ & $-\frac{80}{69}$ & $\frac{12}{23}$ \\
\hline 28 & -4 & 4 & 0 & 5 & 6 & -3 & $\frac{9}{23}$ & 0 & 0 & 0 & $\frac{151}{184}$ & $-\frac{149}{92}$ & $-\frac{8}{23}$ & $\frac{25}{184}$ \\
\hline 29 & -4 & 4 & 8 & -1 & -6 & 7 & $\frac{4}{69}$ & 0 & 0 & 0 & $-\frac{5}{92}$ & $-\frac{17}{46}$ & $-\frac{1}{69}$ & $\frac{1}{92}$ \\
\hline 30 & -4 & 4 & 16 & -1 & -6 & -1 & 0 & $\frac{1}{23}$ & 0 & $\underline{1}$ & 104 & $-\frac{32}{42}$ & -64 & $\begin{array}{r}92 \\
\underline{8} \\
\end{array}$ \\
\hline 31 & -4 & 5 & 0 & 0 & 11 & -4 & 8 & 0 & 0 & 69 & $\begin{array}{r}23 \\
85 \\
\end{array}$ & $\begin{array}{r}23 \\
79 \\
\end{array}$ & $\begin{array}{r}69 \\
2 \\
\end{array}$ & ${ }^{23}{ }_{17}$ \\
\hline 32 & -4 & 5 & 0 & 6 & -1 & 2 & $\begin{array}{r}23 \\
27 \\
\end{array}$ & 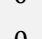 & -1 & 0 & $\overline{92} 63$ & $45^{46}$ & $7^{23}$ & $\begin{array}{l}92 \\
45\end{array}$ \\
\hline 33 & -4 & 6 & & & & 2 & $\begin{array}{c}184 \\
4\end{array}$ & 0 & $-\overline{184}$ & 0 & $5^{368}$ & $\begin{array}{l}184 \\
25\end{array}$ & $\begin{array}{l}46 \\
5\end{array}$ & $-\frac{368}{368}$ \\
\hline 34 & & 0 & 8 & -5 & -8 & 11 & $\frac{7}{207}$ & 0 & 0 & $-\frac{1}{207}$ & $\frac{5}{92}$ & $-\overline{46}$ & $\frac{5}{69}$ & $-\frac{15}{92}$ \\
\hline 34 & -4 & 10 & 0 & -7 & 0 & 9 & $\frac{1}{69}$ & 0 & 0 & $\frac{1}{69}$ & $-\frac{1}{23}$ & $\frac{1}{23}$ & $\frac{1}{69}$ & 0 \\
\hline 35 & -4 & 10 & 0 & 5 & 0 & -3 & $-\frac{1}{69}$ & 0 & $\frac{3}{23}$ & $\frac{7}{60}$ & 407 & $-\frac{283}{-}$ & $-\frac{15}{-}$ & $\underline{1}$ \\
\hline 36 & -4 & 11 & 8 & -6 & -7 & 6 & 0 & 0 & 0 & $\begin{array}{l}69 \\
1 \\
\end{array}$ & $\begin{array}{l}92 \\
-15\end{array}$ & $110^{46}$ & $\begin{array}{r}23 \\
-16 \\
\end{array}$ & $\begin{array}{l}92 \\
30 \\
\end{array}$ \\
\hline 37 & -4 & 15 & 0 & -8 & 1 & 4 & 0 & & & ${ }^{23} 3$ & $19^{23}$ & $\begin{array}{l}23 \\
7\end{array}$ & $2^{23}$ & $\overline{23}_{15}$ \\
\hline 38 & -3 & & & & & & 1 & 0 & 0 & $-\overline{23}$ & $\overline{92}$ & $\overline{46}_{33}$ & $\overline{23}_{1}$ & $-\frac{15}{92}$ \\
\hline 38 & -2 & 2 & 5 & 2 & -4 & 6 & $\frac{1}{23}$ & 0 & 0 & 0 & $-\frac{53}{736}$ & $-\frac{35}{368}$ & $-\frac{1}{92}$ & $\frac{29}{736}$ \\
\hline 39 & -3 & 3 & -3 & 3 & 13 & -5 & $\frac{6}{23}$ & 0 & 0 & 0 & $\frac{185}{184}$ & $-\frac{199}{92}$ & $-\frac{13}{23}$ & $\frac{55}{184}$ \\
\hline 40 & -3 & 3 & 5 & -3 & 1 & 5 & $\frac{8}{207}$ & 0 & 0 & 1 & $-\frac{5}{4}$ & $\underline{4}^{20}$ & $-\frac{2}{-2}$ & 7 \\
\hline 41 & -3 & 4 & -3 & -2 & 18 & -6 & $-\frac{16}{60}$ & 0 & 0 & $\begin{array}{l}207 \\
1 \\
\end{array}$ & $49^{46}$ & $\begin{array}{l}23 \\
-51\end{array}$ & $\begin{array}{r}23 \\
-20 \\
\end{array}$ & $\begin{array}{l}46 \\
-1\end{array}$ \\
\hline 42 & -3 & 4 & -3 & 4 & 6 & 0 & $\frac{9}{92}^{69}$ & 0 & $-\frac{1}{0}$ & $\begin{array}{l}69 \\
0\end{array}$ & $\begin{array}{l}46 \\
-71 \\
\end{array}$ & $\underline{133}^{23}$ & $\underline{21}^{69}$ & $\begin{array}{r}46 \\
-7 \\
\end{array}$ \\
\hline 43 & -3 & 4 & 5 & -2 & -6 & 10 & $\frac{1}{60}$ & 0 & $0^{92}$ & 0 & $\underline{13}^{736}$ & $\begin{array}{l}368 \\
-103 \\
\end{array}$ & $\begin{array}{l}92 \\
-1\end{array}$ & $\begin{array}{r}32 \\
-21 \\
\end{array}$ \\
\hline 44 & -3 & 4 & 13 & -2 & -6 & 2 & $\begin{array}{l}69 \\
0\end{array}$ & 0 & 0 & 1 & $\begin{array}{l}736 \\
18 \\
\end{array}$ & $\underline{52}^{368}$ & $\begin{array}{r}276 \\
-16 \\
\end{array}$ & $10^{736}$ \\
\hline 45 & -3 & 6 & -3 & 0 & 4 & 4 & $\frac{3}{92}$ & 0 & & $\begin{array}{l}69 \\
0\end{array}$ & $\begin{array}{l}23 \\
-65 \\
\end{array}$ & $\begin{array}{l}23 \\
67 \\
\end{array}$ & $\begin{array}{r}69 \\
-1 \\
\end{array}$ & $\begin{array}{l}23 \\
41 \\
\end{array}$ \\
\hline 46 & -3 & 9 & 5 & -3 & -5 & 5 & 0 & 0 & $\begin{array}{l}92 \\
0\end{array}$ & 0 & $\begin{array}{l}{ }^{736} \\
-1^{136}\end{array}$ & $\begin{array}{l}368 \\
\frac{5}{-}\end{array}$ & $0^{92}$ & 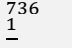 \\
\hline 47 & -3 & 13 & -3 & -5 & 3 & 3 & 0 & 0 & $\frac{2}{20}$ & 0 & $7^{4}$ & $\begin{array}{l}2 \\
103 \\
\end{array}$ & 57 & $\begin{array}{l}4 \\
-479\end{array}$ \\
\hline 48 & -3 & 14 & 5 & -4 & -4 & 0 & $-\frac{1}{60}$ & 0 & $\begin{array}{l}23 \\
2 \\
-\end{array}$ & 7 & $\begin{array}{l}184 \\
503 \\
\end{array}$ & $\begin{array}{l}{ }^{92} \\
-1177\end{array}$ & $\begin{array}{l}23 \\
14 \\
\end{array}$ & $\begin{array}{r}184 \\
-\quad-\frac{13}{}\end{array}$ \\
\hline 49 & -2 & 0 & 2 & 5 & -2 & 5 & $\underline{3}^{69}$ & 0 & 23 & 69 & ${ }^{92}{ }_{57}$ & $27^{46}$ & $\begin{array}{c}23 \\
5\end{array}$ & $\begin{array}{r}4 \\
-\quad 7 \\
\end{array}$ \\
\hline 50 & -2 & 2 & -6 & 1 & 20 & -7 & $\begin{array}{l}92 \\
4 \\
\end{array}$ & 0 & 0 & 0 & $25 \overline{736}$ & $\overline{368}$ & $\overline{92}{ }_{24}$ & ${ }_{18}^{-\overline{736}}$ \\
\hline 50 & 2 & 2 & & 1 & 20 & & $\overline{23}$ & 0 & 0 & 0 & $\frac{5}{23}$ & $-\frac{0}{23}$ & $-\frac{23}{23}$ & $\frac{7}{23}$ \\
\hline 51 & -2 & 2 & 2 & 1 & -4 & 9 & $\frac{1}{92}$ & 0 & 0 & 0 & $\frac{1}{184}$ & & $-\frac{3}{46}$ & $\frac{9}{184}$ \\
\hline 52 & -2 & 2 & 10 & 1 & -4 & 1 & 0 & 0 & 0 & 0 & 1 & 0 & 0 & 0 \\
\hline 53 & -2 & 3 & -6 & 2 & 13 & -2 & $\frac{3}{46}$ & 0 & $-\frac{1}{46}$ & 0 & $-\frac{3}{368}$ & $\frac{33}{184}$ & $\frac{11}{46}$ & $-\frac{101}{368}$ \\
\hline 54 & -2 & 3 & 2 & -4 & 1 & 8 & $\frac{2}{207}$ & 0 & $0^{40}$ & 1 & $\begin{array}{r}368 \\
-\quad-7 \\
\end{array}$ & $\begin{array}{l}184 \\
-3 \\
\end{array}$ & $\begin{array}{ll}46 \\
-\end{array}$ & $\underline{31}^{368}$ \\
\hline 55 & -2 & 3 & 2 & 8 & 1 & -4 & $\begin{array}{l}207 \\
0\end{array}$ & $\underline{1}$ & 0 & 207 & $\begin{array}{l}368 \\
801^{368} \\
\end{array}$ & $\begin{array}{r}184 \\
-603 \\
\end{array}$ & $\begin{array}{r}138 \\
-\frac{9}{-}\end{array}$ & $\begin{array}{l}368 \\
-9 \\
\end{array}$ \\
\hline 56 & -3 & 18 & -3 & -6 & 4 & -2 & $-\frac{1}{0}$ & $\begin{array}{l}23 \\
0\end{array}$ & $-\underline{14}$ & 7 & $\begin{array}{r}368 \\
245 \\
\end{array}$ & $\begin{array}{r}184 \\
-715 \\
\end{array}$ & $\begin{array}{r}46 \\
-534 \\
\end{array}$ & $\begin{array}{r}\begin{array}{r}368 \\
2215\end{array} \\
\end{array}$ \\
\hline 57 & -2 & 5 & 2 & 4 & -1 & 0 & 69 & 0 & ${ }^{23}$ & 69 & $\begin{array}{l}92 \\
21\end{array}$ & $\begin{array}{r}46 \\
15\end{array}$ & $1_{1}^{23}$ & $\begin{array}{l}92 \\
3^{92}\end{array}$ \\
\hline 58 & -2 & 12 & 2 & -1 & -2 & -1 & 0 & 0 & 0 & $\begin{array}{l}0 \\
7\end{array}$ & $\begin{array}{l}16 \\
227\end{array}$ & $\begin{array}{r}8 \\
-625 \\
\end{array}$ & $\begin{array}{l}2 \\
78\end{array}$ & $\begin{array}{l}\overline{16} \\
11\end{array}$ \\
\hline 58 & -2 & 12 & 2 & -1 & -2 & -1 & $-\frac{1}{69}$ & 0 & $\frac{2}{23}$ & $\overline{69}$ & $\overline{92}$ & $-\frac{\overline{46}}{66}$ & $\begin{array}{l}-\overline{23} \\
19\end{array}$ & $\frac{\pi}{4}$ \\
\hline 59 & -1 & -4 & -1 & 12 & 2 & 0 & $\frac{27}{368}$ & 0 & $\frac{1}{368}$ & 0 & $\frac{3}{46}$ & $-\frac{6}{23}$ & $-\frac{19}{92}$ & $\frac{3}{46}$ \\
\hline 60 & -1 & 1 & -9 & -1 & 27 & -9 & $\frac{8}{69}$ & 0 & 0 & $-\frac{1}{69}$ & $\frac{51}{46}$ & -3 & $-\frac{124}{69}$ & $\frac{73}{46}$ \\
\hline 61 & -1 & 1 & -1 & -1 & 3 & 7 & $\frac{1}{138}$ & 0 & 0 & 0 & $-\frac{5}{736}$ & $-\frac{17}{260}$ & $-\frac{35}{27}$ & $\frac{93}{726}$ \\
\hline 62 & -1 & 1 & -1 & 11 & 3 & -5 & -11853 & $\underline{1}$ & -209 & 27 & $\underline{93}^{736}$ & $\begin{array}{r}368 \\
1620 \\
\end{array}$ & $7^{229^{276}}$ & $\begin{array}{l}736 \\
-459 \\
\end{array}$ \\
\hline & -1 & 1 & & & & & 5336 & 23 & 5336 & $\begin{array}{l}2668 \\
1\end{array}$ & $\begin{array}{r}29 \\
28 \\
\end{array}$ & $\begin{array}{c}29 \\
-52 \\
\end{array}$ & $\begin{array}{l}116 \\
16\end{array}$ & $\begin{array}{l}667 \\
10\end{array}$ \\
\hline 63 & -1 & 1 & 7 & -1 & 3 & -1 & 0 & 0 & 0 & $-\frac{1}{69}$ & & $-\frac{\overline{23}}{23}$ & $\overline{69}$ & \\
\hline 64 & -1 & 2 & -9 & 0 & 20 & -4 & $\frac{1}{23}$ & 0 & $-\frac{1}{23}$ & 0 & $\frac{3}{46}$ & $-\frac{2}{23}$ & 0 & $-\frac{3}{46}$ \\
\hline 65 & -1 & 2 & -1 & 0 & -4 & 12 & $\frac{1}{368}$ & 0 & $-\frac{1}{368}$ & 0 & $\frac{3}{736}$ & $-\frac{25}{268}$ & 0 & $-\frac{3}{77}$ \\
\hline 66 & -1 & 2 & -1 & 6 & 8 & -6 & 0 & $\frac{1}{20}$ & 0 & 0 & $\underline{159}$ & $-\underline{129}$ & 7 & -39 \\
\hline 67 & -1 & 2 & 7 & 0 & -4 & 4 & 0 & $\begin{array}{l}23 \\
0\end{array}$ & 0 & 0 & $\begin{array}{l}184 \\
0\end{array}$ & $1^{92}$ & $\begin{array}{l}23 \\
0\end{array}$ & $0^{184}$ \\
\hline 68 & -1 & 3 & -1 & -5 & 1 & 11 & $\frac{1}{111}$ & 0 & 0 & 1 & $-\frac{9}{70}$ & $\underline{11}$ & $\underline{13}$ & $-\frac{31}{37}$ \\
\hline 69 & -1 & 3 & -1 & 7 & 1 & -1 & $\begin{array}{l}414 \\
0\end{array}$ & 0 & 1 & $\begin{array}{l}207 \\
0\end{array}$ & $\underline{927}^{736}$ & $\begin{array}{l}368 \\
-909 \\
\end{array}$ & $\begin{array}{l}276 \\
-81 \\
-1\end{array}$ & $4^{741^{736}}$ \\
\hline 70 & -1 & 4 & 7 & -4 & -6 & 8 & 0 & 0 & $\begin{array}{l}46 \\
0\end{array}$ & $\underline{1}$ & $\begin{array}{l}736 \\
--5\end{array}$ & $\underline{29}^{368}$ & $\begin{array}{r}92 \\
-16 \\
\end{array}$ & $\begin{array}{l}736 \\
10 \\
\underline{-}\end{array}$ \\
\hline 71 & -1 & 8 & -1 & -6 & 2 & 6 & 0 & 0 & 0 & $\begin{array}{l}69 \\
-1 \\
-1\end{array}$ & $5^{23}$ & $\begin{array}{l}23 \\
-3\end{array}$ & $\begin{array}{r}69 \\
-7 \\
\end{array}$ & $\begin{array}{r}23 \\
59 \\
\end{array}$ \\
\hline 72 & 0 & -1 & -4 & 2 & 5 & 6 & 1 & 0 & 1 & $0^{23}$ & $\begin{array}{l}184 \\
-\quad 1 \\
\end{array}$ & $\begin{array}{r}92 \\
-5 \\
\end{array}$ & $\begin{array}{r}23 \\
-3 \\
\end{array}$ & $\begin{array}{l}184 \\
21 \\
\end{array}$ \\
\hline 12 & & & & & & & & & & 0 & & & $-\overline{46}$ & \\
\hline
\end{tabular}




\begin{tabular}{|c|c|c|c|c|c|c|c|c|c|c|c|c|c|c|}
\hline 73 & 0 & 0 & -4 & -3 & 10 & 5 & 1 & 0 & 0 & -1 & 1 & $-\frac{7}{-}$ & $-\frac{4}{-}$ & 31 \\
\hline 74 & 0 & 0 & -4 & -0 & 10 & -7 & 207 & 1 & $-\frac{1}{1}$ & 207 & $\begin{array}{l}184 \\
-63 \\
\end{array}$ & 153 & $36{ }^{23}$ & $\begin{array}{l}184 \\
-225 \\
\end{array}$ \\
\hline 14 & 0 & 0 & -4 & 9 & 10 & -1 & 0 & $\overline{23}$ & $-\overline{23}$ & & $\overline{184}$ & $\overline{92}$ & $\overline{23}$ & $-\frac{-184}{184}$ \\
\hline 75 & 0 & 0 & 4 & 3 & -2 & 3 & 0 & 0 & 0 & 0 & $\frac{1}{8}$ & $\frac{1}{4}$ & 0 & $-\frac{1}{8}$ \\
\hline 76 & 0 & 1 & -12 & -2 & 27 & -6 & $\frac{2}{69}$ & 0 & $-\frac{2}{23}$ & $-\frac{1}{69}$ & $\frac{7}{46}$ & $-\frac{13}{23}$ & $-\frac{52}{69}$ & $\frac{31}{46}$ \\
\hline 77 & 0 & 1 & -4 & -2 & 3 & 10 & $\frac{1}{552}$ & 0 & $-\frac{1}{184}$ & 0 & $-\frac{3}{736}$ & $-\frac{7}{368}$ & $-\frac{17}{276}$ & $\frac{51}{736}$ \\
\hline 78 & 0 & 1 & -4 & 4 & 15 & -8 & 0 & $\frac{1}{23}$ & 0 & 0 & $-\frac{47}{07}$ & $\frac{85}{468}$ & $3^{20}$ & $\begin{array}{l}736 \\
-77 \\
-\end{array}$ \\
\hline 79 & 0 & 1 & 4 & -2 & 3 & 2 & 0 & 0 & 0 & $-\frac{1}{9}$ & $5^{92}$ & $-\frac{6}{-66}$ & $\begin{array}{l}23 \\
16 \\
\underline{1}\end{array}$ & $\begin{array}{r}92 \\
-10 \\
\end{array}$ \\
\hline 80 & 0 & 2 & 4 & -1 & -4 & 7 & 0 & 0 & 0 & $0^{69}$ & ${ }^{23} 1$ & $3^{323}$ & 69 & $\underline{1}^{23}$ \\
\hline 81 & 0 & 6 & -4 & -3 & 4 & 5 & 0 & 0 & $\frac{1}{2}$ & 0 & $\begin{array}{r}8 \\
-1 \\
\end{array}$ & $\begin{array}{l}4 \\
10 \\
\underline{-1}\end{array}$ & 17 & $\begin{array}{l}8 \\
-17\end{array}$ \\
\hline 82 & 0 & 7 & 4 & -2 & -3 & 2 & 0 & 0 & $\begin{array}{l}23 \\
0\end{array}$ & 0 & $2^{23}$ & $\begin{array}{l}23 \\
-8\end{array}$ & $\begin{array}{c}23 \\
0\end{array}$ & $1^{23}$ \\
\hline 83 & 0 & 11 & -4 & -4 & 5 & 0 & 0 & 0 & $-\frac{8}{22}$ & 0 & $\underline{147}$ & -321 & $-\frac{182}{2}$ & $\begin{array}{l}1 \\
\underline{705} \\
\end{array}$ \\
\hline 84 & 0 & 14 & 4 & -7 & -4 & 1 & $-\frac{1}{69}$ & 0 & $\underline{2}^{23}$ & 88 & $\begin{array}{l}92 \\
-841 \\
\end{array}$ & $\begin{array}{r}46 \\
1727 \\
\end{array}$ & $\begin{array}{r}23 \\
-418 \\
\end{array}$ & $\begin{array}{r}92 \\
2389 \\
\end{array}$ \\
\hline 85 & 1 & -2 & 1 & 6 & 0 & 2 & 0 & 0 & $\begin{array}{l}23 \\
0\end{array}$ & $\begin{array}{l}69 \\
0\end{array}$ & $\underline{7}^{92}$ & $\begin{array}{l}46 \\
-5 \\
\end{array}$ & $-1^{23}$ & $\underline{1}^{92}$ \\
\hline 86 & 1 & -1 & -7 & 7 & 17 & -9 & 0 & $\underline{1}$ & -2 & 0 & $32 \quad 285$ & $\begin{array}{r}16 \\
435 \\
\end{array}$ & $65^{4}$ & $\begin{array}{l}32 \\
-411\end{array}$ \\
\hline 87 & 1 & -1 & 1 & 1 & 5 & 1 & 0 & 23 & 23 & 0 & 184 & $\overline{92}$ & $\overline{23}$ & $\frac{-184}{184}$ \\
\hline 88 & 1 & 0 & -7 & 1 & J & & $\begin{array}{l}0 \\
1\end{array}$ & 0 & 0 & 0 & $\frac{1}{4}$ & $-\frac{1}{2}$ & 0 & $-\frac{1}{4}$ \\
\hline 88 & & 0 & -7 & -4 & 10 & 8 & $\frac{\overline{8}}{828}$ & 0 & $-\frac{1}{92}$ & $-\frac{1}{207}$ & $\frac{3}{736}$ & $-\frac{25}{269}$ & $-\frac{47}{276}$ & $\frac{133}{776}$ \\
\hline 89 & 1 & 0 & -7 & 2 & 22 & -10 & 0 & $\frac{1}{23}$ & 0 & 0 & $-\frac{81}{46}$ & $\frac{123}{23}$ & $\frac{76}{23}$ & $-\frac{119}{46}$ \\
\hline 90 & 1 & 0 & 1 & 2 & -2 & 6 & 0 & 0 & 0 & 0 & $-\frac{3}{32}$ & $\frac{9}{16}$ & $\frac{1}{4}$ & $-\frac{5}{32}$ \\
\hline 91 & 1 & 0 & 9 & 2 & -2 & -2 & 0 & $\frac{1}{23}$ & 0 & 0 & $-\frac{6}{23}$ & $-\frac{84}{23}$ & $-\frac{16}{23}$ & $-\frac{2}{23}$ \\
\hline 92 & 1 & 5 & 1 & 1 & -1 & 1 & 0 & 0 & 0 & 0 & $\frac{3}{2}$ & -6 & -2 & \\
\hline 93 & 2 & -4 & -2 & 9 & 2 & 1 & 0 & 0 & $\frac{1}{92}$ & 0 & $\frac{121}{368}$ & $-\frac{141}{184}$ & $-\frac{13}{23}$ & $\frac{23}{369}$ \\
\hline 94 & 2 & -2 & -10 & 5 & 24 & -11 & 0 & $\underline{1}$ & $-\frac{4}{20}$ & 0 & $\begin{array}{r}368 \\
-119\end{array}$ & 175 & $100^{23}$ & $\begin{array}{l}368 \\
-167\end{array}$ \\
\hline 95 & 2 & -2 & -2 & -1 & 12 & -1 & 0 & $\begin{array}{l}23 \\
0\end{array}$ & $0^{23}$ & 1 & $13^{46}$ & ${ }_{-17}^{23}$ & ${ }^{23} 16$ & $\begin{array}{r}46 \\
-\frac{3}{4}\end{array}$ \\
\hline 96 & 2 & -2 & -2 & 5 & 0 & 5 & 0 & 0 & $-\frac{1}{1}$ & 69 & 4661 & $\begin{array}{l}167^{23} \\
\end{array}$ & $29^{69}$ & $\begin{array}{r}46 \\
-163 \\
\end{array}$ \\
\hline 97 & 2 & -2 & 6 & 5 & 0 & -3 & 0 & 1 & $0^{92}$ & 0 & $\begin{array}{r}736 \\
-29\end{array}$ & $\begin{array}{l}368 \\
\underline{8}\end{array}$ & 92 & $\begin{array}{r}736 \\
-\quad 2 \\
-\quad 2\end{array}$ \\
\hline 98 & 2 & -1 & -10 & 0 & 29 & -12 & 0 & $\begin{array}{l}23 \\
1 \\
\end{array}$ & 0 & 1 & $\begin{array}{r}23 \\
-67 \\
\end{array}$ & $\begin{array}{l}23 \\
210 \\
\end{array}$ & $152^{23}$ & $\begin{array}{r}23 \\
-133 \\
\end{array}$ \\
\hline 99 & 2 & -1 & -2 & 0 & 5 & 4 & 0 & $\begin{array}{l}23 \\
0\end{array}$ & 0 & $\begin{array}{l}23 \\
0\end{array}$ & $\begin{array}{r}23 \\
-1 \\
\end{array}$ & $\frac{3}{2}^{23}$ & $\underline{1}^{23}$ & $\begin{array}{r}23 \\
-7^{23}\end{array}$ \\
\hline 100 & 2 & -1 & 6 & 0 & 5 & -4 & 0 & $\frac{1}{2}$ & 0 & $\underline{1}$ & $\begin{array}{r}16 \\
-44 \\
\end{array}$ & $\begin{array}{l}8 \\
72 \\
\end{array}$ & ${ }^{2}-32$ & $\underline{28}^{16}$ \\
\hline 101 & 2 & 1 & -2 & -4 & 3 & 8 & 97 & $\begin{array}{l}23 \\
0\end{array}$ & 5 & $\begin{array}{r}23 \\
7 \\
\end{array}$ & $\begin{array}{r}23 \\
- \\
-\end{array}$ & $\begin{array}{l}23 \\
-897\end{array}$ & $\begin{array}{r}23 \\
-175 \\
\end{array}$ & ${ }^{23} 2$ \\
\hline 102 & 2 & 1 & -2 & 8 & 3 & -4 & $\begin{array}{l}116 \\
-12033 \\
\end{array}$ & 1 & $\begin{array}{l}1044 \\
-277 \\
\end{array}$ & $\begin{array}{l}174 \\
-711 \\
\end{array}$ & $\underline{90}^{261}$ & $\begin{array}{r}29 \\
6291 \\
\end{array}$ & $\begin{array}{r}58 \\
1161 \\
\end{array}$ & $\begin{array}{l}-\overline{29} \\
162\end{array}$ \\
\hline 103 & 3 & -3 & -13 & 3 & 31 & -13 & 2668 & $\begin{array}{c}23 \\
1\end{array}$ & $\begin{array}{r}2668 \\
-\quad 8\end{array}$ & 1334 & $\begin{array}{l}\overline{29} \\
-157\end{array}$ & $\begin{array}{r}29 \\
227 \\
\end{array}$ & $\begin{array}{c}\overline{58} \\
124\end{array}$ & $\overline{{ }^{667}}{ }_{215}$ \\
\hline 104 & 3 & -3 & -5 & 3 & 7 & 10 & 0 & $\overline{23}$ & $\begin{array}{c}-\overline{23} \\
1\end{array}$ & 0 & $\begin{array}{l}46 \\
53\end{array}$ & $\begin{array}{r}23 \\
127 \\
\end{array}$ & $\begin{array}{c}\overline{23} \\
35\end{array}$ & $\begin{array}{r}-\overline{46} \\
211\end{array}$ \\
\hline 105 & 3 & -3 & 3 & 3 & 7 & & 0 & 0 & $-\overline{46}$ & 0 & $\begin{array}{r}-\overline{736} \\
52\end{array}$ & $\begin{array}{r}368 \\
100 \\
\end{array}$ & $\overline{92}{ }_{16}$ & $-\frac{736}{2}$ \\
\hline 106 & 3 & -2 & - & . & $\gamma$ & -5 & 0 & $\frac{1}{23}$ & 0 & 0 & $-\frac{32}{23}$ & $\begin{array}{l}\overline{23} \\
47\end{array}$ & $-\overline{23}$ & $-\frac{2}{23}$ \\
\hline & & -2 & -5 & -2 & 12 & 2 & 0 & 0 & 0 & $\frac{1}{69}$ & $-\frac{17}{184}$ & $\overline{92}$ & $\frac{53}{69}$ & $-\frac{127}{184}$ \\
\hline 107 & 3 & -2 & 3 & 4 & 0 & 0 & 0 & 0 & 0 & 0 & $\frac{1}{2}$ & -2 & 0 & \\
\hline 108 & 3 & -1 & -5 & -1 & 5 & 7 & 0 & 0 & $\frac{1}{46}$ & 0 & $\frac{7}{736}$ & $\frac{11}{368}$ & $\frac{11}{92}$ & $-\frac{111}{736}$ \\
\hline 109 & 3 & 0 & 3 & 0 & -2 & 4 & 0 & 0 & 0 & 0 & $\frac{1}{2}$ & -2 & 0 & $-\frac{1}{2}$ \\
\hline 110 & 3 & 4 & -5 & -2 & 6 & 2 & 0 & 0 & $-\frac{4}{23}$ & 0 & $\frac{55}{184}$ & $-\frac{137}{92}$ & $-\frac{45}{2}$ & $\frac{337}{109}$ \\
\hline 111 & 3 & 7 & 3 & -5 & -3 & 3 & 0 & 0 & $0^{23}$ & $\underline{9}$ & $\begin{array}{l}184 \\
-20\end{array}$ & $\underline{116}^{92}$ & $\begin{array}{r}23 \\
-144 \\
\end{array}$ & $\begin{array}{l}184 \\
178 \\
\end{array}$ \\
\hline 112 & 3 & 11 & -5 & -7 & 5 & 1 & 0 & 0 & $-\frac{32}{2}$ & $\begin{array}{l}{ }^{23} \\
-\frac{27}{27}\end{array}$ & $3^{23}$ & $\begin{array}{l}23 \\
-438 \\
\end{array}$ & $\begin{array}{r}23 \\
-848 \\
\end{array}$ & $\begin{array}{r}23 \\
861 \\
\end{array}$ \\
\hline 113 & 4 & -4 & -16 & 1 & 38 & -15 & 0 & $\frac{1}{23}$ & $-\frac{16}{23}$ & $\begin{array}{r}23 \\
-\frac{1}{22}\end{array}$ & $-\frac{90}{22}$ & $2^{244^{23}}$ & $\underline{96}^{23}$ & $\begin{array}{l}23 \\
-82\end{array}$ \\
\hline 114 & 4 & -4 & -8 & 1 & 14 & 1 & 0 & $\begin{array}{l}23 \\
0\end{array}$ & $-\frac{1}{23}$ & $0^{23}$ & $\begin{array}{r}23 \\
-\frac{15}{194}\end{array}$ & $\begin{array}{l}23 \\
\frac{29}{92}\end{array}$ & $\begin{array}{l}\frac{23}{6} \\
\frac{6}{22}\end{array}$ & $\begin{array}{r}23 \\
-25 \\
\end{array}$ \\
\hline 115 & 4 & -4 & 0 & 1 & 14 & -7 & 0 & $\frac{1}{23}$ & $0^{23}$ & $-\frac{1}{23}$ & $\begin{array}{l}\frac{184}{20} \\
-\frac{60}{20}\end{array}$ & $\begin{array}{l}92 \\
\frac{128}{23}\end{array}$ & $\begin{array}{l}23 \\
0\end{array}$ & $\begin{array}{l}\frac{184}{22} \\
-\frac{32}{20}\end{array}$ \\
\hline 116 & 4 & -4 & 0 & 7 & 2 & -1 & 0 & $\begin{array}{l}23 \\
0\end{array}$ & 0 & $0^{23}$ & $0^{23}$ & $\begin{array}{l}23 \\
0\end{array}$ & 1 & $0^{23}$ \\
\hline 117 & 4 & -3 & 0 & 2 & 7 & -2 & 0 & 0 & 0 & 0 & 0 & 0 & 0 & 1 \\
\hline 118 & 4 & -2 & 0 & 3 & 0 & 3 & 0 & 0 & 0 & 0 & $\frac{1}{2}$ & -2 & -1 & $\frac{1}{2}$ \\
\hline 119 & 4 & 3 & 0 & 2 & 1 & -2 & $-\frac{1}{6}$ & 0 & $\frac{2}{20}$ & $\frac{7}{9}$ & $-\frac{601}{0}$ & 1031 & $\frac{198}{20}$ & -25 \\
\hline 120 & 5 & -6 & -3 & 10 & 4 & -2 & $0^{69}$ & 0 & $\begin{array}{l}{ }^{23} \\
-\frac{1}{-}\end{array}$ & $\begin{array}{l}69 \\
0\end{array}$ & $\begin{array}{r}92 \\
-405 \\
\end{array}$ & $\begin{array}{r}46 \\
783 \\
\end{array}$ & $\begin{array}{r}23 \\
231 \\
\end{array}$ & $\begin{array}{r}4 \\
-675 \\
\end{array}$ \\
\hline 121 & 5 & -5 & -11 & -1 & 21 & -1 & 0 & 0 & $\begin{array}{r}23 \\
-2 \\
\end{array}$ & -1 & $\begin{array}{r}736 \\
-\quad-13 \\
\end{array}$ & $\begin{array}{l}368 \\
11 \\
\underline{-1}\end{array}$ & $\begin{array}{l}92 \\
-17\end{array}$ & $\underline{77}^{736}$ \\
\hline 122 & 5 & -5 & -3 & 5 & 9 & -3 & 0 & 0 & $0^{23}$ & $0^{69}$ & $\begin{array}{c}184 \\
-1^{184}\end{array}$ & $\begin{array}{l}92 \\
2\end{array}$ & $2^{69}$ & $\begin{array}{l}184 \\
-1\end{array}$ \\
\hline 123 & 5 & -4 & -3 & 0 & 14 & -4 & 0 & 0 & 0 & $-\frac{1}{2}$ & $\begin{array}{r}2 \\
-8 \\
\end{array}$ & $\underline{28}$ & $\underline{16}$ & $\underline{16}^{2}$ \\
\hline 124 & 5 & -4 & -3 & 6 & 2 & 2 & 0 & 0 & 1 & 23 & $\begin{array}{l}4^{23} \\
405^{2}\end{array}$ & $\begin{array}{l}23 \\
-783 \\
\end{array}$ & $\begin{array}{l}23 \\
-139\end{array}$ & $\begin{array}{l}23 \\
675 \\
\end{array}$ \\
\hline 125 & 5 & 1 & -3 & 5 & 3 & -3 & $-\frac{1}{-1}$ & 0 & ${ }^{23}{ }_{6}$ & $\begin{array}{l}0 \\
7\end{array}$ & $\begin{array}{r}736 \\
148\end{array}$ & $493^{368}$ & $246^{92}$ & $\begin{array}{c}736 \\
\quad 209\end{array}$ \\
\hline 126 & 6 & -6 & -6 & 3 & 16 & -5 & $-\overline{69}$ & 0 & $-\overline{23}$ & $\overline{69}$ & $-\frac{23}{23}$ & $\overline{23}$ & $\overline{23}$ & $-\frac{23}{23}$ \\
\hline $\begin{array}{l}120 \\
127\end{array}$ & & -5 & -6 & 3 & 10 & & & 0 & 0 & 0 & -1 & $4_{415}$ & & $\begin{array}{l}-2 \\
491\end{array}$ \\
\hline 127 & 6 & -5 & -6 & 4 & 9 & 0 & 0 & 0 & $\frac{2}{23}$ & 0 & $\frac{21}{368}$ & $-\frac{415}{184}$ & $-\frac{5}{46}$ & $\frac{491}{368}$ \\
\hline 128 & 6 & -3 & -6 & 0 & 7 & 4 & 0 & 0 & $-\frac{2}{23}$ & 0 & $-\frac{37}{368}$ & $\frac{47}{184}$ & $\frac{1}{46}$ & $\frac{61}{368}$ \\
\hline
\end{tabular}




\begin{tabular}{|c|c|c|c|c|c|c|c|c|c|c|c|c|c|c|}
\hline 129 & 6 & 0 & 2 & -3 & -2 & 5 & 0 & 0 & 0 & 3 & $\underline{1}$ & 8 & -48 & $\underline{44}$ \\
\hline & & 4 & & & & & & & $-\frac{16}{16}$ & $\begin{array}{l}23 \\
-9\end{array}$ & $\begin{array}{l}23 \\
49 \\
\end{array}$ & $\overline{23}$ & $\begin{array}{r}23 \\
-312 \\
\end{array}$ & $\begin{array}{l}\overline{23} \\
625 \\
\end{array}$ \\
\hline 130 & 6 & 4 & -6 & -5 & 6 & 3 & 0 & 0 & $-\overline{23}$ & $-\overline{23}$ & & -7 & $-\overline{23}$ & $\overline{46}$ \\
\hline 131 & 6 & 5 & 2 & -4 & -1 & 0 & $-\frac{1}{69}$ & 0 & $\frac{2}{23}$ & & $-\frac{361}{92}$ & $\frac{335}{46}$ & $\frac{446}{23}$ & $-\frac{1883}{92}$ \\
\hline 132 & 6 & 9 & -6 & -6 & 7 & -2 & $-\frac{1}{69}$ & 0 & 130 & $\frac{250}{6}$ & $-\frac{1465}{0}$ & 3839 & 3102 & $-\frac{12347}{}$ \\
\hline 133 & 7 & -7 & -9 & 1 & 23 & -7 & 0 & 0 & 23 & $\begin{array}{l}1 \\
1\end{array}$ & $\begin{array}{r}92 \\
-\end{array}$ & $\begin{array}{r}46 \\
156 \\
\end{array}$ & $\begin{array}{r}23 \\
168 \\
\end{array}$ & $\begin{array}{r}92 \\
-108 \\
\end{array}$ \\
\hline 134 & 7 & -6 & -9 & 2 & 16 & -2 & 0 & 0 & 4 & 23 & $\begin{array}{r}23 \\
129^{2} \\
\end{array}$ & 23 & 23.47 & $215^{23}$ \\
\hline 125 & & & 1 & 2 & 10 & -2 & 0 & 0 & 23 & 0 & $\begin{array}{l}184 \\
81\end{array}$ & 54 & $\begin{array}{l}23 \\
108\end{array}$ & $\begin{array}{c}184 \\
27\end{array}$ \\
\hline 135 & 7 & -6 & -1 & 8 & 4 & -4 & 0 & $\overline{23}$ & 0 & 0 & $-\frac{1}{46}$ & $\overline{23}$ & $-\overline{23}$ & $-\overline{46}$ \\
\hline 136 & 7 & -4 & -1 & 4 & 2 & 0 & 0 & 0 & 0 & 0 & $-\frac{3}{2}$ & 6 & 4 & $-\frac{3}{2}$ \\
\hline 137 & 7 & 3 & -1 & -1 & 1 & -1 & $-\frac{1}{69}$ & 0 & $\frac{2}{23}$ & $\frac{7}{69}$ & $-\frac{601}{92}$ & $\frac{1031}{46}$ & $\frac{750}{32}$ & $-\frac{133}{4}$ \\
\hline 138 & 8 & -8 & -4 & 11 & 6 & -5 & 0 & $\frac{1}{2}$ & $\underline{4}$ & 69 & $81^{92}$ & $\begin{array}{r}46 \\
-567 \\
\end{array}$ & -270 & $567^{4}$ \\
\hline 139 & 8 & -7 & -12 & 0 & 23 & -4 & 0 & 0 & $\begin{array}{r}23 \\
8 \\
\end{array}$ & 1 & $\begin{array}{l}184 \\
-3\end{array}$ & $\begin{array}{r}92 \\
-103 \\
\end{array}$ & $\begin{array}{r}23 \\
-18 \\
\end{array}$ & $\begin{array}{l}184 \\
-33 \\
\end{array}$ \\
\hline 140 & 8 & -7 & -4 & 6 & 11 & -6 & 0 & $\underline{1}$ & $\begin{array}{l}23 \\
0\end{array}$ & $\begin{array}{l}23 \\
0\end{array}$ & -6 & $\begin{array}{r}46 \\
-84 \\
\end{array}$ & $\begin{array}{r}23 \\
-200 \\
\end{array}$ & $\underline{21}^{92}$ \\
\hline 141 & 8 & -6 & -4 & 7 & 4 & -1 & 0 & 0 & $-\frac{4}{-}$ & 0 & $\begin{array}{r}23 \\
-405 \\
\end{array}$ & $\underline{783}^{23}$ & $162^{23}$ & $\begin{array}{l}23 \\
-675 \\
\end{array}$ \\
\hline 142 & 9 & -9 & -7 & 9 & 13 & -7 & 0 & $\frac{1}{1}$ & $\frac{8}{8}^{23}$ & 0 & ${\underline{243^{184}}}$ & $\begin{array}{l}92 \\
-675 \\
\end{array}$ & $\begin{array}{l}23 \\
-432 \\
\end{array}$ & $27^{184}$ \\
\hline 143 & 9 & -8 & -7 & 4 & 18 & -8 & 0 & $\begin{array}{l}23 \\
1 \\
\end{array}$ & $\begin{array}{l}23 \\
0\end{array}$ & 0 & $\begin{array}{l}92 \\
\underline{40} \\
\end{array}$ & $\begin{array}{r}46 \\
-268 \\
\end{array}$ & $\begin{array}{r}23 \\
-384 \\
\end{array}$ & $\begin{array}{l}4 \\
136 \\
\end{array}$ \\
\hline 144 & 9 & -3 & -7 & -3 & 7 & 5 & 0 & 0 & $-\frac{8}{-}$ & $-\frac{3}{-}$ & $\frac{5}{5}$ & $\begin{array}{r}23 \\
-61 \\
\end{array}$ & $\begin{array}{r}23 \\
-88 \\
\end{array}$ & $\begin{array}{r}23 \\
17 \\
\end{array}$ \\
\hline 145 & 9 & -2 & 1 & -2 & 0 & 2 & 0 & 0 & $0^{23}$ & $-\frac{9}{23}$ & $\begin{array}{l}92 \\
-26\end{array}$ & $\underline{68}^{46}$ & $\underline{144}^{23}$ & $\begin{array}{l}486 \\
--1\end{array}$ \\
\hline 146 & 9 & 2 & -7 & -4 & 8 & 0 & 0 & 0 & 64 & $2^{23}$ & $\begin{array}{r}23 \\
-78 \\
\end{array}$ & $\begin{array}{l}23 \\
528 \\
\end{array}$ & $\begin{array}{l}23 \\
1024 \\
\end{array}$ & -1014 \\
\hline 147 & 10 & -4 & -2 & 1 & 2 & 1 & 0 & 0 & 23 & 23 & 23 & 23 & 23 & 23 \\
\hline 148 & 10 & -10 & -10 & 7 & 20 & -9 & 0 & $\underline{1}$ & 16 & 0 & 255 & 591 & -664 & 579 \\
\hline 149 & 10 & -9 & -10 & 2 & 25 & -10 & 0 & $\begin{array}{l}23 \\
1 \\
\end{array}$ & 23 & 3 & $\begin{array}{l}46 \\
108\end{array}$ & 23 & $\begin{array}{c}23 \\
704\end{array}$ & 46 \\
\hline 150 & 11 & -11 & -13 & 5 & 27 & -11 & 0 & $\begin{array}{l}23 \\
1 \\
\end{array}$ & 32 & 23 & $\begin{array}{r}23 \\
215 \\
\end{array}$ & $\begin{array}{r}-24 \\
-914 \\
\end{array}$ & $\begin{array}{r}23 \\
-944 \\
\end{array}$ & $\begin{array}{l}10 \\
443\end{array}$ \\
\hline 151 & 11 & -8 & -5 & 8 & 6 & -4 & $-\frac{1}{-}$ & 23 & $\begin{array}{l}23 \\
18\end{array}$ & 7 & $\begin{array}{l}23 \\
623 \\
\end{array}$ & $\begin{array}{c}23 \\
1363\end{array}$ & $\begin{array}{r}23 \\
726 \\
\end{array}$ & $\begin{array}{l}23 \\
1189\end{array}$ \\
\hline 152 & 11 & & & 0 & 0 & -4 & 69 & 1 & $\begin{array}{l}23 \\
64\end{array}$ & $\begin{array}{l}69 \\
3\end{array}$ & 92 & $\begin{array}{c}\frac{46}{1276} \\
\end{array}$ & $\begin{array}{l}-\overline{23} \\
1184\end{array}$ & $\begin{array}{r}92 \\
518\end{array}$ \\
\hline 152 & 12 & -12 & -16 & 3 & 34 & -13 & 0 & $\overline{23}$ & $\overline{23}$ & $\overline{23}$ & 14 & $-\frac{23}{23}$ & $-\frac{23}{216}$ & 23 \\
\hline 153 & 12 & -5 & -8 & -2 & 9 & 2 & 0 & 0 & $\frac{33}{23}$ & $\frac{9}{23}$ & $\frac{17}{23}$ & & & $-\frac{274}{23}$ \\
\hline 154 & 13 & -6 & -3 & 2 & 4 & -2 & $-\frac{1}{69}$ & 0 & $\frac{2}{23}$ & $\frac{7}{69}$ & $\frac{1055}{92}$ & $-\frac{2281}{46}$ & $-\frac{1458}{23}$ & $\frac{155}{4}$ \\
\hline 155 & 14 & -8 & -6 & 5 & 6 & -3 & $-\frac{1}{6}$ & 0 & 66 & $\frac{7}{7}$ & 1811 & $-\frac{3577}{7}$ & -1842 & 3997 \\
\hline 156 & 16 & -6 & -4 & -1 & 4 & -1 & $-\frac{1}{-}$ & 0 & 2 & -236 & $\begin{array}{r}92 \\
3431 \\
\end{array}$ & $\begin{array}{r}46 \\
7681 \\
\end{array}$ & $\begin{array}{r}23 \\
-4578 \\
\end{array}$ & $\begin{array}{r}92 \\
13717 \\
\end{array}$ \\
\hline
\end{tabular}

